\title{
Studies on monolayer and langmuir-blodgett films of schiff- base complex 1,2-bis(3-thiophene imine) ethylene
}

\author{
Keke Zou ${ }^{1,2}$, Kai Xu ${ }^{3}$, Tiesheng $\mathrm{Li}^{1, \mathrm{a}}$, Hui Liu ${ }^{1}$, Wenjian $\mathrm{Xu}^{1}$, and Yangjie $\mathrm{Wu}^{1}$ \\ ${ }^{1}$ The College of Chemistry and Molecular Engineering, Zhengzhou University, Zhengzhou 450052, P. R. China. \\ ${ }^{2}$ Pingdingshan Hydrology and Water Resources Survey Bureau of Henan Province, Pingdingshan 467000, P. R. \\ China. \\ ${ }^{3}$ Hydrology and Water Resources Bureau of Henan Province, Zhengzhou 450003, P. R. China.
}

\begin{abstract}
The Langmuir- Blodgett (LB) technique makes it possible to prepare a thin film with a controlled thickness at the molecular level and a well-defined molecular orientation. In this work, 1,2-bis(3-thiophene imine) ethylene (B3TIE) were synthesized by reacting benzaldehyde and Ethylenediamine and were characterized by MS, IR, ${ }^{1} \mathrm{HNMR},{ }^{13} \mathrm{CNMR}$. B3TIE monolayer and LB films were prepared through LB technology and characterized by Water contact angle analysis, FT-IR, UV-vis, CV and AFM. An electrochemical study of B3TIE monolayer on ITO was carried out. The cyclic voltammetric data revealed that the reduction on ITO was irreversible. Meanwhile, layer structure and surface morphology of LB films were also explored. The results show that monolayer can be transferred onto the hydrophilic substrates to form regular monolayer and multilayers, in which molecular orientation can be observed from the surface morphology.
\end{abstract}

Keywords: LB films; thiophene imine; schiff-Base complex.

\section{Introduction}

Schiff-base Complex is a class of imine or benzophenone imine tomb group (-Rc $=\mathrm{N}-)$ compounds, widely used in medicine, catalysis, analysis, corrosion, discoloration of light-induced fields, materials, etc[1]. In recent years, it has been is a striking object of study. Currently, the study of Schiff base compounds focused on the synthesis of compounds, characterization, properties of complexes and photochromic, electrochemistry etc[2,3]. Meanwhile, Some Schiff bases have thermochromic and photochromic properties in the solid state[4]. They are also used as model systems in studies of biological macromolecules and catalytic reactions[5,6]. However, LB films film-forming performance research is relatively small.

Langmuir-Blodgett films, which have the advantage of homogeneous structure, special physical and chemistry properties, easy fabrication, easy characterization, has received much attention in recently years. Moreover, the Langmuir- Blodgett (LB) technique makes it possible to prepare a thin film with a controlled thickness at the molecular level and a well-defined molecular orientation ${ }^{[7,8]}$ and has been studied extensively for photolithography [9-13] in which it can not only overcome the weakness of spin-coat films[14,15], in which molecules are distributed randomly, but also be expected to improve the resolution and the sensitivity of the resist effectively [16,17].

${ }^{a}$ Corresponding author : 13619891880@163.com 
In this paper, 1,2-bis(3-thiophene imine) ethylene (B3TIE) were synthesized by reacting benzaldehyde and Ethylenediamine shown in Scheme 1 and were characterized by MS, IR, ${ }^{1} \mathrm{HNMR}$, ${ }^{13}$ CNMR. Behaviors of the Langmuir monolayer at air/water interface and their LB films were investigated by water contact angle analysis, FT-IR, UV-vis, cyclic voltammetry, and Atomic force microscopy.

\section{Experimental}

\subsection{Synthesis of 1, 2-bis(3-thiophene imine) ethylene (B3TIE)}

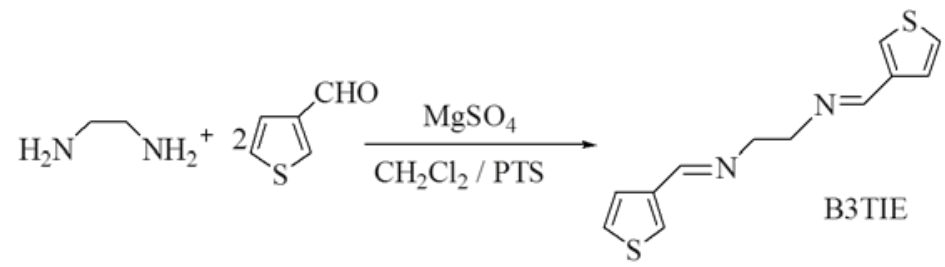

Scheme.1 Synthesis and structure of B3TIE

B3TIE were synthesized by reacting benzaldehyde and Ethylenediamine and were characterized by ${ }^{1} \mathrm{HNMR},{ }^{13} \mathrm{CNMR}, \mathrm{IR}, \mathrm{MS} .{ }^{1} \mathrm{HNMR}\left(400 \mathrm{MHz}, \mathrm{CDCl}_{3}\right): \delta(\mathrm{ppm})=8.29(2 \mathrm{H}, \mathrm{s},-\mathrm{CH}=\mathrm{N}-), 7.54(4 \mathrm{H}, \mathrm{m}$,

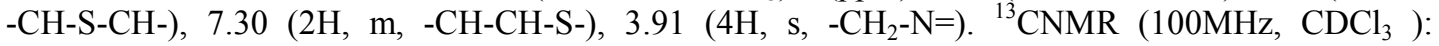
$\delta(\mathrm{ppm})=156.0,139.42,127.49,125.37,124.68,60.68$. IR (KBr, Pellet): 3047, 2919, 2848, 1711, 1638, $1420,825,714 \mathrm{~cm}^{-1}$. MS: $248.9[\mathrm{M}+\mathrm{H}]^{+}$.

\subsection{Measurement}

Infrared spectra were obtained by a Bruker Vector 22. UV-visible absorption spectrum was carried out on Lambda 35 UV-Visible spectrophotometer (Perkin Elmer Inc. USA). Deep UV irradiation was achieved with EX250 UV light source (Honya Schott Ltd., Japan). ${ }^{1} \mathrm{H}$ NMR was measured with NMR DPX-400 (Bruker,German) in $\mathrm{CDCl}_{3}$ and DMSO-d6. Surface pressure-Area isotherm $(\pi-\mathrm{A})$ measurements were carried out on a computer-controlled KSV-5000-3 instrument (KSV Instruments, Helsinki, Finland). Deionized water with resistivity of $18.2 \mathrm{M} \Omega . \mathrm{cm}$ (MILI-Q gradient MILIPORECo., USA) was used for a subphase. The polymer was dissolved in chloroform $\left(\mathrm{CHCl}_{3}\right)$ at a concentration of $0.5 \mathrm{mg} / \mathrm{ml}$ and the solution was spread on the water surface by a micrometric syringe. Waiting for about $30 \mathrm{mins}$ to let the solvent evaporate out, the monolayer compression started at a compression rate of $5 \mathrm{~mm} / \mathrm{min}$. Quartz, glass, $\mathrm{CaF}_{2}$ and silicon wafers used for LB films deposition were prepared by boiling concentrated $\mathrm{HNO}_{3}(60 \%)$ and $\mathrm{H}_{2} \mathrm{SO}_{4}(98 \%)(2: 1)$ solutions, then washed with deionized water to give hydrophilic surface.

\section{Results and discussion}

\subsection{Behavior of the Langmuir monolayer and LB films}

B3TIE was dissolved in dichloromethane formulated to solution of $0.5 \mathrm{mg} / \mathrm{mL}$, then, Solution containing B3TIE was spread over a water surface to measure surface pressure $(\pi)$-area $(A)$ isotherms at $25^{\circ} \mathrm{C}$ (Fig. 1a). Fig.1 a show that the curve of B3TIE is consistent with the standard curve changes in the state, but collapse pressure is not high, this may be due to hydrophobic chain of B3TIE is shorter. The results also proved that Schiff-Base Complex with short hydrophobic chain had the lower collapse pressure [18]. From the experimental resuIts it can be seen that monolayers of B3TIE could 
be transferred onto hydrophilic glass slides as a Z-type film under surface pressure of $8 \sim 16 \mathrm{mN} / \mathrm{m}$ by Langmuir-Blodgett technique, indicating that the LB films were uniform and stable $[19,21]$. The isotherm showed the molecular area is $\sim 48 \AA^{2} / \mathrm{molecule}$ at $12 \mathrm{mN} / \mathrm{m}$. This is calculate that the content of B3TIE Langmuir monolayer in the substrate $\left(35 \times 10 \mathrm{~mm}^{2}\right)$ was $1.65 \times 10^{-10} \mathrm{~mol} / \mathrm{cm}^{2}$.
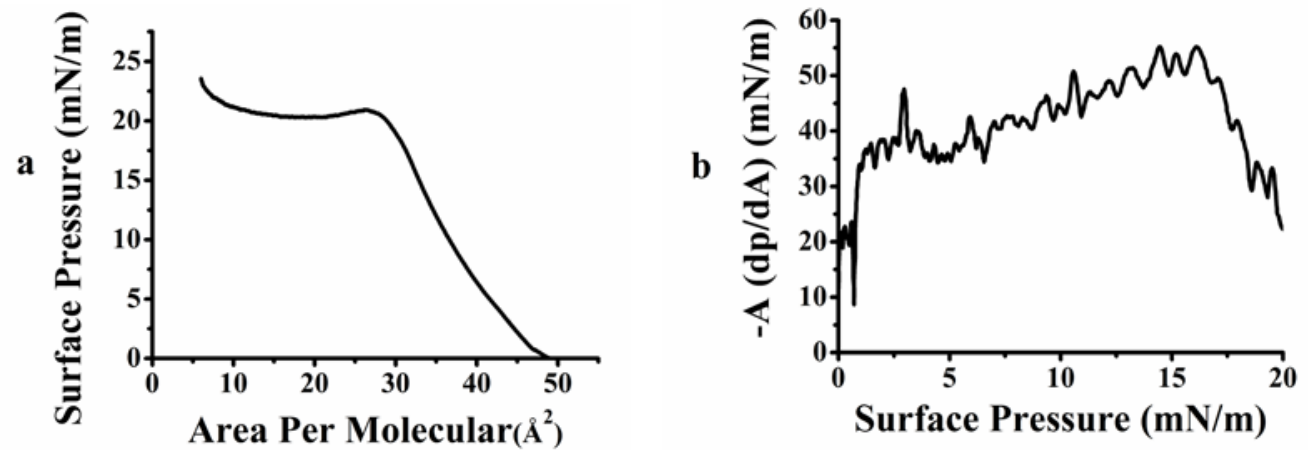

Figure 1. $\pi$-A isotherm (a) and Es- $\pi$ isotherm (b) of B3TIE

In order to obtain optimum conditions for LB films deposition of B3TIE monolayer, the static elasticity (Es)-surface pressure $(\pi)$ isotherm of B3TIE monolayer was studied (Fig.1b). The Es- $\pi$ isotherm was much plainer, more sensitive and better to describe the monolayer state than $\pi$-A isotherm, because it could give more detailed information on molecular interaction and motion. High static elasticity modulus and relatively stable area is more suitable for LB films deposition surface pressure area [20]. The static elasticity (Es)-surface pressure $(\pi)$ isotherm of B3TIE monolayer, is shown in Figure 1b, the static elasticity isotherm indicate $8 \sim 16 \mathrm{mN} / \mathrm{m}$ was optimum surface pressure for LB films deposition from water surface. Next, the surface pressure of $12 \mathrm{mN} / \mathrm{m}$ as an example is used to explore the characteristics of monolayer and LB films. The monolayer and LB films were analyzed by using water contact angle analysis, FT-IR, UV-vis, cyclic voltammetry, and Atomic force microscopy.

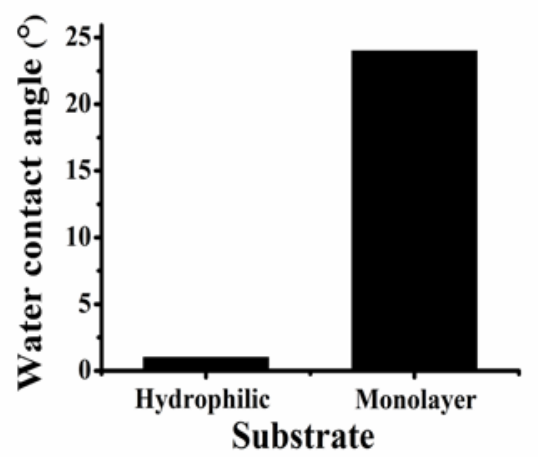

Figure 2. Water contact angle histogram of B3TIE monolayer on silicon

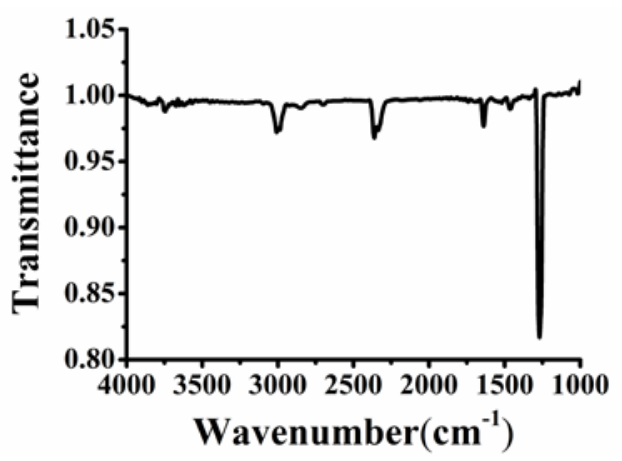

Figure 3. IR spectrum of B3TIE LB film deposited onto $\mathrm{CaF}$

Water contact angle can reflect surface wettability which was caused by either a chemical gradient or a gradient in the topographical surface structure[22]. Water contact angles on modied solid surfaces are shown in Fig. 2. The water contact angle of hydrophilic substrates was $3^{\circ}$, which shows that the glass substrate has good hydrophilic activity. In the case of B3TIE monolayer, the contact angle increased from $3^{\circ}$ to $24^{\circ}$, indicating that the surface configuration has a significant change [23]. These results proved B3TIE couid be transferred to the substrate form LB fiims.

IR spectrum can detect functional stretching vibration and bending vibration, provides a powerful means to identify the unknown organic compound. LB films of B3TIE deposited onto CaF (2 layers) was studied by ATR-IR (fig.4). The absorption band at about $1640 \mathrm{~cm}^{-1}$ was representative of the 
imine stretching vibration peak, appeared in the $1250 \mathrm{~cm}^{-1} \mathrm{~S}-\mathrm{H}$ key on the thiophene ring, stretching vibration peak at $1450 \mathrm{~cm}^{-1}$ a thiophene skeleton stretching vibration absorption peak, the saturated $2850 \mathrm{~cm}^{-1} \mathrm{C}-\mathrm{H}$ stretching vibration, appeared in the $3020 \mathrm{~cm}^{-1} \mathrm{C}-\mathrm{H}$ key on the thiophene ring stretching vibration, indicating that B3TIE couid be transferred to the substrate form LB fiims at room temperature.

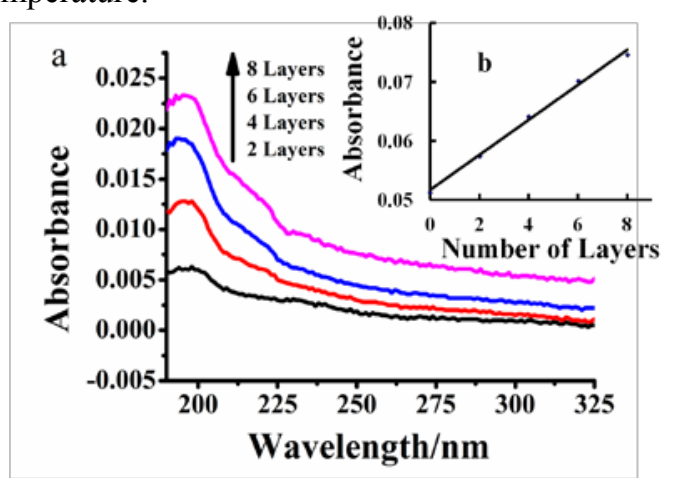

Figure 4. (a) UV-vis spectra of B3TIE Multilayer LB films; (b) Plot of the absorbance at $196 \mathrm{~nm}$ as a function of the number of the bilayers.

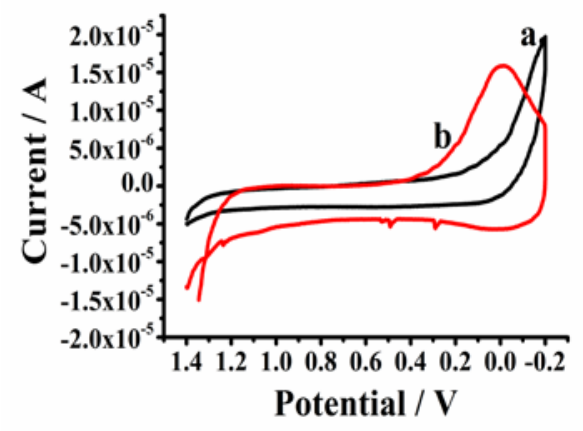

Figure 5. Cyclic voltammograms of B3TIE monolayer transferred onto ITO

The UV-vis spectra of B3TIE Multilayer LB films are shown in Figure 3a, Figure 3b is Plot of the absorbance at $196 \mathrm{~nm}$ as a function of the number of the bilayers. The film exhibits nearly linear growth $(296 \mathrm{~nm})$, and the maximal absorbance at $196 \mathrm{~nm}$ was proportional to the number of layers deposited. The linear relationship between the maximal absorbance and the number of layers in the UV-vis spectra indicates that the regular homogeneous deposition occurred and B3TIE monolayer could be transferred onto substrate to form LB films with The amount of transfer almost unity.

\subsection{Electrochemical properties}

The electrochemical can detect some special functional groups redox signaling. The Cyclic voltammograms of B3TIE grafted onto ITO glass and hydrophilic ITO were measured electrochemistry properties (Fig.3), Figure $3 \mathrm{a}$ is a cyclic voltammogram of hydrophilic ITO, Figure $3 \mathrm{~b}$ is a cyclic voltammogram of B3TIE monolayer. The cyclic voltammograms were conducted in a three-electrode system, B3TIE grafted onto ITO glass was used as the working electrode, a Pt wire as the counter electrode, and $\mathrm{KCl}(0.1 \mathrm{M})$ as the reference electrode. The redox peaks are displayed between -0.3 to $+1.5 \mathrm{~V}$ at room temperature, and the sweep rate was set at $100 \mathrm{mV} / \mathrm{s}$. The $\mathrm{CV}$ curves showed that there is only one reduction peak observed in the -0.2 to $0.3 \mathrm{~V}$ potential range. In addition, the anodic half cycle does not have any peak, indicating that the electroreduction is irreversible ${ }^{[24]}$. By contrast, even for hydrophilic ITO, with the most positive reduction potential, there was no peak observed at scan rates $100 \mathrm{mV} / \mathrm{s}$, indicating that hydrophilic treatment effect is better, surface clean without impurities. Through the analysis of the sample, we learned that reduction peaks is amine absorption peak of B3TIE monolayer [25]. At the same time, Figure 3 shows that B3TIE monolayer film successfully transferred to the substrate.

\subsection{Morphology of the Langmuir monolayer and LB films}

AFM is a scanning probe technique that can provide three-dimensional imaging at a nanometer scale resolution of surface structures and it allows a quantitative analysis of sample morphology. Figure. 5 is the AFM image of (a) silicon wafer, (b) B3TIE monolayer and (c) B3TIE 20 layers LB films. Their roughness are $0.635,0.546$ and $1.104 \mathrm{~nm}$, respectively. Fig.5b showed the typical AFM images of B3TIE LB monolayer, shows that there is a single layer film of B3TIE tightly packed orderly, and show a certain orientation. Fig.5c showed the 20 layers LB films. The films presented regular 
"waviness", It indicated that each layer could be transferred uniform and a condensed LB films were formed. Figure.5 shows that LB films successfully transferred to the hydrophilic substrates, and multilayer films have a layered structure and good surface morphology.
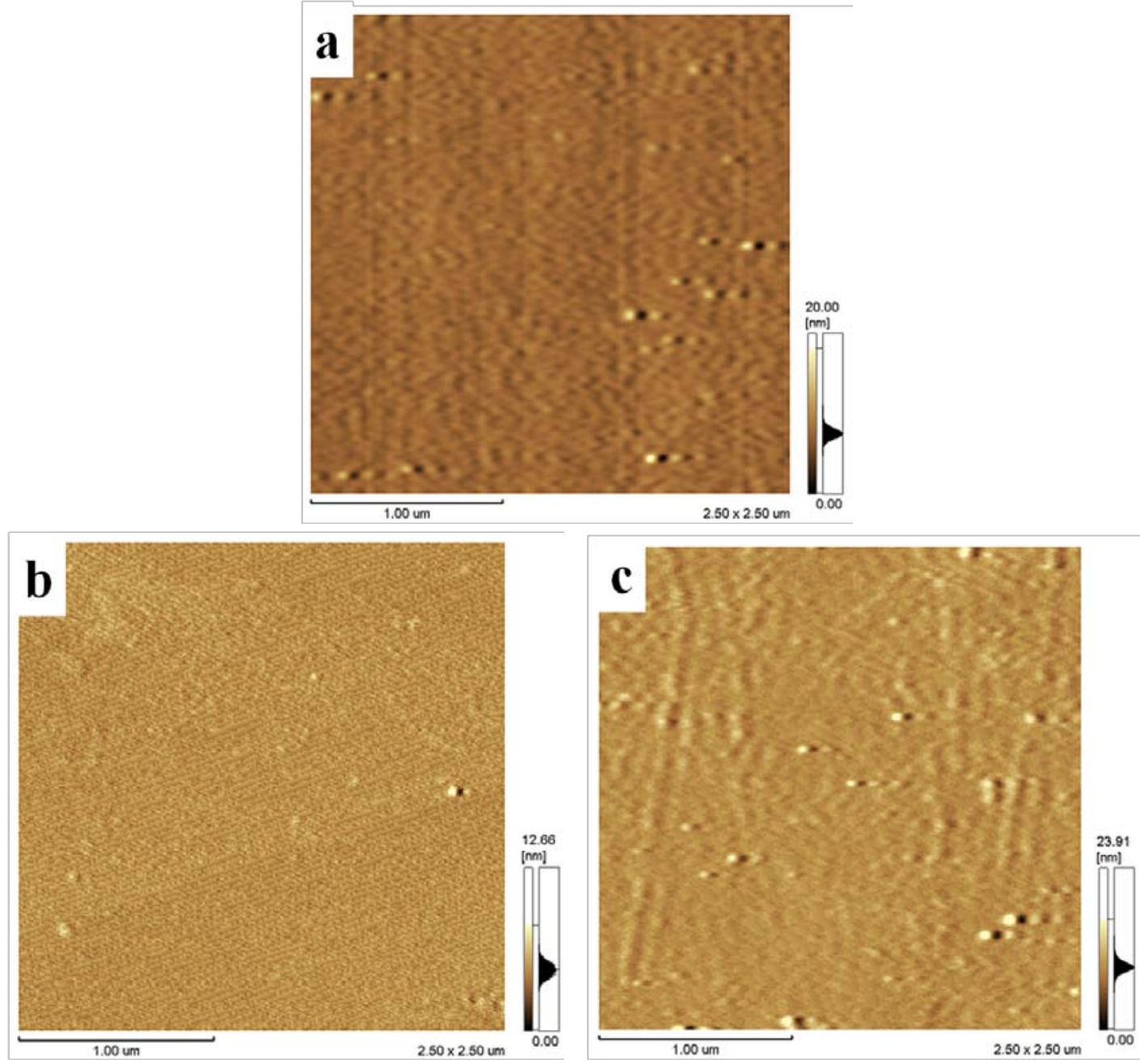

Figure 6. AFM images of silicon (a), B3TIE monolayer (b) and B3TIE 20-layer LB films (c)

\section{Equations and mathematics}

1,2-bis(3-thiophene imine) ethylene (B3TIE) were prepared and characterized. B3TIE monolayer and LB films were characterized and can be transferred on to solid substrates by the Langmuir-Blodgett (LB) method. Through the water contact Angle and the infrared characterization, known B3TIE successfully transferred to solid substrate to form the LB film. Through UV characterization, shows B3TIE can be layered homogeneous transferred to the substrate. At the same time, the cyclic voltammograms of monolayer film characterization, the results show that the B3TIE successfully transferred to the substrate. Morphology of the B3TIE monolayer and multilayer were also investigated with AFM method. The images obtained showed that B3TIE monolayer and multilayer had homogeneous topographic, indicating that B3TIE monolayer could be transferred uniformly and a condensed LB films were formed. 


\section{Ackonwledgement}

The authors thank prof. Luyuan Mao, College of Materials Science and Engineering, Zhengzhou University, for the use of AFM. This work is partially Supported by the National Natural Science Foundation of China (No. 20973157) and the Research and Development Foundation of Zhengzhou (094SGZG23056).

\section{References}

1. J. Sagiv, J. Am. Chem. Soc., 102, 9-95 (1980)

2. A.A. Isse, A. Gennaro, E. Vianello, Electrochemical Acta, 42, 2065-2071 (1980)

3. J. Casaszar, J. Morray, O. Herczeg, Acta Phys. Chem, 31, 717 (1985)

4. M.E. Kletskii, A.A. Millov, A.V. Metelitsa, M.I. Knyazhansky, J. Photochem. Photobiol. A, 110, 267 (1997)

5. D. Chen, A.E. Martel, Inorg. Chem, 26, 1026 (1987)

6. D.E. Hamilton, R.S. Drago, A. Zonbeck, J. Am. Chem. Soc., 109, 374 (1987).

7. K.B. Blodgett, J. Am. Chem. Soc., 57, 1007-1022 (1935)

8. K.B. Blodgett, I. Langmuir, Phys. Rev, 51, 964-982 (1937)

9. A. Aoki, M. Nakaya, T. Miyashita, Chemistry Letters, 8, 667-668 (1996)

10. Y.Z. Guo, F. Feng, T. Miyashita, Chemistry Letters, 12, 1269-1270 (1998)

11. Y.Z. Guo, F. Feng, T. Miyashita, Macromolecules, 32, 1115-1118 (1999)

12. A. Barraud, Thin Solid Films, 99, 317-321 (1983)

13. R.V. Sudiwala, C. Cheng, E.G. Wilson, D.N. Batchelder, Thin Solid Films, 210, 452-454 (1992)

14. R. Hui, Z.W. Wang, S. Yick, R. Maric, D. Ghosh, Journal of Power Sources, 172, 840-844 (2007)

15. P. Jasinski, S. Molin, M. Gazda, V. Petrovsky, H.U. Anderson, Journal of Power Sources, 194, 10-15 (2009)

16. X.D. Li, A. Aoki, T. Miyashita, Macromolecules, 30, 2194-2196 (1997)

17. H. Takano, M. Fujihira, Thin Solid Films, 273, 312-316 (1996)

18. T.S. Li, M. Masaya, M. Tokuji, High school chemistry, 4, 544-546 (2006)

19. S.F. Pang, Z.F. Ye, C. Li, Y.Q. Liang, Journal of Colloid and Interface Science, 240, 480-486 (2001)

20. Y.H. Shen, B. Xia, A.J. Xie, Y.F. Tang, Colloids and Surfaces A., 252, 21-25(2005)

21. N. Zhao, F. Wang, M.L. Zhou, T.S. Li, H. Liu, W.J. Xu, Y.J. Wu, Chinese Journal of Catalysis, 34, 1583-1588 (2013)

22. J.S. Sharp, D.J. Farmer, J. Kelly, Langmuir, 27, 9367 (2011)

23. N. Zhao,T.S. Li, Z. Zhai, J.J. Qiu, W.J. Xu, H. Liu, Y.J. Wu, ChemCatChem, 44, 1-10 (2013)

24. K. Polat, M. Uçar, M.L. Aksu, H. Ünver, Can. J. Chem., 82, 1150-1156 (2004) 